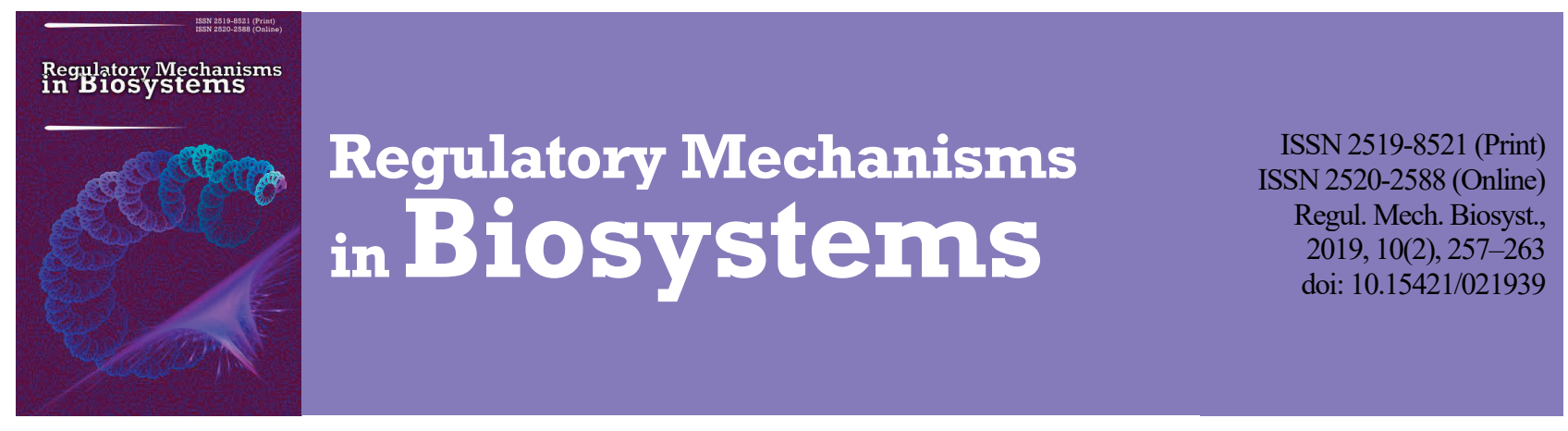

\title{
Detection of biogenic polyamines in blood of patients with breast cancer
}

\author{
M. P. Prylutskyi*, N. M. Bilko*, N. F. Starodub** \\ *National University of "Kyiv-Mohyla Academy", Kyiv, Ukraine \\ **National University of Life and Environmental Sciences of Ukraine, Kyiv, Ukraine
}

Article info

Received 02.02.2019

Received in revised form 05.03.2019

Accepted 09.03.2019

National University

of "Kyiv-Mohyla Academy",

Grygorij Skovoroda st., 2,

Kyiv, 04070, Ukraine.

Tel. $+38-044-463-70-67$.

E-mail:

prilutskiy.maxim@gmail.com

National University of Life

and Environmental Sciences

of Ukraine, Herojiv Oborony st.,

15, Kyiv, 03041, Ukraine.

Tel. $+38-044-531-36-52$

E-mail: rectorat@mubip.edu.ua

\section{Prylutskyi, M. P., Bilko, N. M., \& Starodub, N. F. (2019). Detection of biogenic polyamines in blood of patients with breast cancer. Regulatory Mechanisms in Biosystems, 10(2), 257-263. doi:10.15421/021939}

The main threat of cancer diseases is their spreading throughout the population of many countries of the world and the complexity of their diagnostics at the early stages. Because of that, search and development of the latest diagnostic methods for oncological diseases which would allow them to be diagnosed more precisely and rapidly is going on. The objective of the study was developing a method for qualitative and quantitative analysis of polyamines as potential tumour markers in blood serum of patients with breast cancer using a newly created immune biosensor based on the effect of surface plasmon resonance (SPR). In the process of the study we used basics of immune analysis, methods of biosensor analysis with preliminary modification of biosensor analytical surface with certain reagents, such as protein A and BSA, for better orientation of the sensitive layer made from antibodies. During the study 30 samples of blood serum were analyzed, 21 of which were obtained from the patients with breast cancer and 9 samples were normal, taken from healthy people and used as controls. Analysis of blood serum samples was made, using previously created calibration curve, based on polyamine solutions in concentrations from $5 \mathrm{ng} / \mathrm{mL}$ to $1 \mu \mathrm{g} / \mathrm{mL}$. It allowed determination of the presence of polyamines in blood samples and approximate concentration of polyamines comparing resonance angle shift in calibration curve and blood samples. According to the obtained results, the concentration of polyamines exceeded their physiological levels and was in the range of $21.3-125.1 \mathrm{ng} / \mathrm{mL}$. The proposed approach allows one to determine the presence and approximate concentrations of polyamines in range from $5 \mathrm{ng} / \mathrm{mL}$ to $1 \mu \mathrm{g} / \mathrm{mL}$ in samples of blood serum of patients with breast cancer which correlates with tumour size and the age of the patients.

Keywords: surface plasmon resonance; biosensors; spermine; tumour markers; transducer; sensogram.

\section{Introduction}

For the early diagnostics of breast cancer, and assessment of efficiency of malignant tumours treatment, and detection of metastases, the methods based on the detection of tumour markers are used. The experience of using tumour markers in diagnostics has shown that their introduction into medical practice significantly increases the efficiency of treatment (Park \& Igarashi, 2013). These markers are metabolites of neoplastic cells, which appear in the case of oncological disease and can be very specific for a tumours of a certain type. In healthy people such compounds are usually absent or present in small amounts. Abnormal expression of genes that occurs in tumour cells leads to alteration of differentiation and proliferation. There may also occur metabolic changes accompanied by the synthesis of certain compounds, which differ qualitatively or quantitatively from those produced by normal cells (Kiran et al., 2018).

All markers are divided into two main groups: those that are produced and secreted in biological fluids exclusively by tumour cells (tumour-associated), and those production and secretion of which are the result of metabolic disorders caused by cancer. They can be specific and non-specific. Concentration of tumour markers depends on many factors, in particular on the properties of the tumour, the treatment performed and the biochemical characteristics of the marker itself. In patients with non-cancerous pathology, the level of such compounds, usually, does not exceed the normal values. However, the non-specific increase of their level may occur. In particular, it can be heightened in case of such pathological processes as inflammatory diseases of the liver, pancreas, lungs, as well as other tissues and organs. In modern oncology practice, research of tumour markers is conducted in order to perform cancer pathology screening, assessment of prevalence of disease (a subsidiary criterion), diagnostics of recurrences and metastases, and evaluation of the effectiveness of treatment. One of such promising markers is polyamines (Moskalets \& Shcherbina, 2011).

Polyamines, such as putrescine, spermidine, and spermine, are present in all living cells. They are important for the growth of eukaryotic cells (Cervelli et al., 2014). These polycations interact with negatively charged molecules, such as DNA, RNA, acid proteins, and phospholipids, and modulate various cellular functions, including macromolecular synthesis. Dysregulation of the level of polyamines leads to pathological conditions, including cancer, inflammation, stroke, renal failure and diabetes. An increase in the concentration of polyamines and enzymes of their synthesis is often associated with tumour growth, and their level in urine and plasma can be used as a diagnostic indicator of cancer (Casero, 2018). Putrescine $\left[\mathrm{NH}_{2}\left(\mathrm{CH}_{2}\right)_{4} \mathrm{NH}_{2}\right]$, spermidine $\left.\left[\mathrm{NH}_{2}\left(\mathrm{CH}_{2}\right)_{4} \mathrm{NH} \quad \mathrm{CH}_{2}\right)_{3} \mathrm{NH}_{2}\right]$, and spermine $\left[\mathrm{NH}_{2}\left(\mathrm{CH}_{2}\right)_{3} \mathrm{NH}\left(\mathrm{CH}_{2}\right)_{4} \mathrm{NH}\left(\mathrm{CH}_{2}\right)_{3} \mathrm{NH}_{2}\right]$ are organic polycations present in all eukaryotes and are essential for cell proliferation (Damiani \& Wallace, 2017). Since their primary and secondary amino groups are protonated at the physiological $\mathrm{pH}$, they electrostatically interact with negatively charged molecules, such as DNA, RNA, proteins, and phospholipids, and regulate the cell cycle during transcription and translation.

Polyamines differ from inorganic cations, such as $\mathrm{Mg}^{2+}$ or $\mathrm{Ca}^{2+}$, because their positive charges are located at large distances in flexible methylene chains, which can participate in hydrophobic interactions. Thus, polyamines enter into stronger and more specific interactions with nucleic acids and acid macromolecules than inorganic cations. Although concentrations of polyamines are usually at millimolar levels in euka- 
ryotic cells, most of them are separated and/or associated with nucleic acids and other negatively charged molecules. Consequently, the concentrations of free polyamines are significantly lower than their total amount in the cell (Soda et al., 2009). Usually, homeostasis of polyamines is supported by complex mechanisms of multipass feedback at the levels of their biosynthesis, catabolism, absorption and excretion (Mandal, 2013). Excessive accumulation of polyamines is usually associated with cell transformation or apoptosis, whereas their reduction/depletion leads to inhibition of cell growth, migration, and embryonic development. Elevated levels of polyamines and their biosynthetic enzymes, namely ornithine decarboxylase (ODC) and S-adenosylmethionine decarboxylase (SAMDC), are often associated with hyperproliferation and oncological processes (Yatsenko, 2014). Activation of the catalytic pathway of polyamines causes increased oxidative stress and may contribute to the ageing and development of pathological conditions, which arise as a result of cell damage (Bae et al., 2018).

\section{Materials and methods}

Materials used for the study were highly specific serums against spermine (Abcam, Cambridge, England) obtained from the Kavetsky Institute of Experimental Pathology, Oncology and Radiobiology (Kyiv, Ukraine). As an analytical device we used a Plasmontest optical biosensor device on the basis of the SPR phenomenon, equipped with a sensor with a resolution of 2048 pixels and connected directly to a computer for the registration and processing of the received signal. The device was developed at the Glushkov Institute of Cybernetics of National Academy of Sciences of Ukraine (Kyiv, Ukraine). Before the experiment we obtained 30 samples of blood serum from the "Alfa-Lab Service" Diagnostic Laboratory (Kharkiv, Ukraine). We divided the received samples into four groups: I group - control group, samples of which were taken from healthy people; II group - test group with samples obtained from patients with breast cancer and size of the tumour less than $2.5 \mathrm{~cm}$, and in age $45-55$ years; III group - test group with samples taken from patients with tumour size more than $2.8 \mathrm{~cm}$ and age range 57-63 years; IV group - with samples obtained from patients with tumour size more than $5 \mathrm{~cm}$ and age $64-70$ years.

Before start the experiment we prepared the surface of imuuno-biosensor on the basis of surface plasmon resonance. Firstly we washed the transducer plate with distilled water and dried under a stream of air. Then the plate was immersed in a freshly prepared solution of "Piranhas" (a mixture of 3:1 concentrated $\mathrm{H}_{2} \mathrm{SO}_{4}$ and $30 \% \mathrm{H}_{2} \mathrm{O}_{2}$ ) for 2 minutes at room temperature. Then the plate was washed again with distilled water. The washed transducer was installed on the prism of the device, and immersion oil was added. The golden surface served as the base for a flow measuring cell with a volume of about $20 \mu \mathrm{L}$.

At the beginning of the experiment a special analytical system was created. We used $0.1 \mathrm{M}$ phosphate-buffered saline (PBS), which was introduced into the measuring cell in a volume of $1 \mathrm{~mL}$ and incubated for $1-2 \mathrm{~min}$ and shifts of the resonance angle was recorded. For the determination of polyamines, a modification of the golden surface was performed using protein A from Staphylococcus aureus (Sigma-Aldrich), which has four binding domains for $\mathrm{Fc}$ fragments of the specific anti-spermine serum. We applied protein A dissolved in PBS at a concentration of $1 \mu \mathrm{g} / \mathrm{mL}$ and incubated it for 20 minutes to orient the future sensitive layer of serum specific antibodies to spermine with $\mathrm{F}(\mathrm{ab})$ side. After a thorough washing of the surface using PBS and recording the value of resonance angle in the cell, we made a solution of serum specific to spermine (Sigma-Aldrich) at a concentration of $100 \mu \mathrm{g} / \mathrm{mL}$ in volume of $20 \mu \mathrm{L}$. Then a solution of bovine serum albumin (BSA) was applied onto a transducer surface in a volume of $20 \mu \mathrm{g}$ at a concentration of $1 \mu \mathrm{g} / \mathrm{mL}$. After 30 minutes, the transducer surface was washed with PBS and shifts of the resonance angle were recorded.

The use of BSA was related to the necessity of blocking possible free places on the surface of the transducer, which could remain after the immobilization of specific monoclonal antibodies. Such preprepared transducer surfaces may be stored (in air-dried room at room temperature) for up to 10 days at a temperature $+2 \ldots+6^{\circ} \mathrm{C}$. After addition of BSA we started to create calibration curve with increasing the con- centrations of spermine in order to determine detection limits of biosensor. We added various $(5-1000 \mathrm{ng} / \mathrm{mL})$ concentrations of spermine and recorded antigen-antibody interactions in real time after each wash. In this case, the resonance angle was initially recorded at the point of insertion into the measuring cell. When the critical angle of a planepolarized light was exceeded, a complete internal reflection occured. The interaction of antigen-specific antibodies with polyamines is recorded according to the alterations of the reflection angle by the type of dependence, which allows monitoring the process of antigen binding with the antibody and causes high sensitivity at determining the polyamine level. After developing the calibration curve, a series of identical experiments were performed, but as a studied sample we used not spermine but different concentrations of blood serum of patients with breast cancer. Results of the experiment were recorded in Plasmon 7.0 program (Glushkov Institute of Cybernetics of National Academy of Sciences, Ukraine).

Duration of each stage of preliminary processing of the transducer surface was within 10-15 minutes at room temperature. At the end of the previous preparation of the transducer surface, the value of the resonance angle in the presence of PBS was recorded. After this, the measuring cell, where the transducer surface was located, was filled with the analyzed solution containing the blood serum, and the value of the resonance angle was determined. During the formation of the immune complexes on the transducer surface, there was recorded a shift of the resonance angle. Changes in the shifts of the angle depended on the number of immune complexes formed on the transducer surface.

All the results were statistically analyzed and presented as $\mathrm{x} \pm \mathrm{SD}$ ( $x \pm$ standard deviation). We determined the differences between control and experimental groups using the Tukey test, where the differences were considered significant at $\mathrm{P}<0.05$ (taking into account the Bonferroni correction).

\section{Results}

We created calibration curve of different concentrations of spermine in order to assess low and high thresholds of the biosensor detection capability. The limits of this calibration curve depended on the concentration of spermine. The lower level of sensitivity was at $5-6 \mathrm{ng} / \mathrm{mL}$. The upper limit of the sensitivity of the biosensor was $1000 \mathrm{ng} / \mathrm{mL}$. According to the obtained data, with each increasing concentration of spermine the shift of resonant angle changed in range 62.8-65.6. The optimum concentrations were in the range 63.1-64.1 degrees at $100 \mathrm{ng} / \mathrm{mL}$. Resonance angle changed by $0.2-0.4$ degrees on average. The obtained data were recorded and analyzed (Fig. 1, 2). Further increase of spermine concentration was not detected by biosensor.

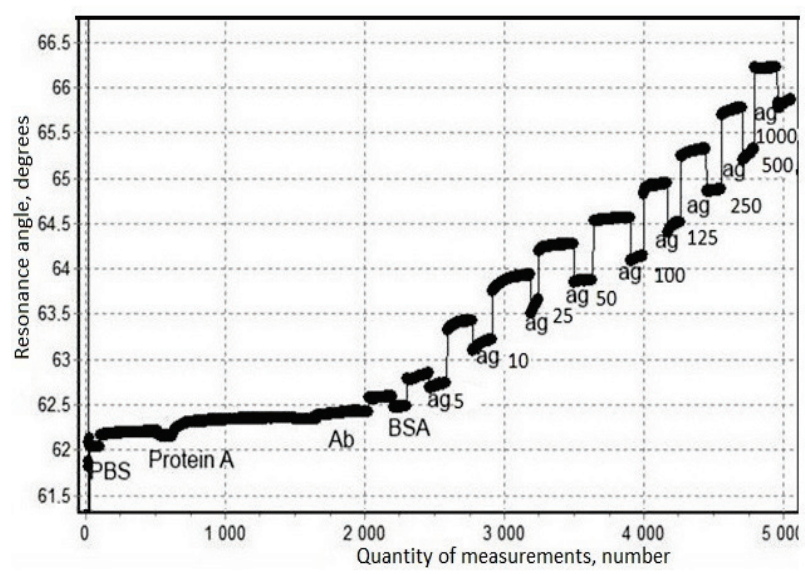

Fig. 1. Sensogram of a biosensor response at different concentrations of sperrmine: PBS - phosphate buffered saline; BSA - bovine serum albumin; $\mathrm{Ab}$ - anti-spermine serum; $\mathrm{Ag}$ - spermine $(5 \mathrm{ng} / \mathrm{mL}$ $1000 \mathrm{ng} / \mathrm{mL})$; sensogram recorded angle shifts per 1 second of real time; number of measurements varied 100-1000 for each solution; all data were recorded in Plasmon 7.0 program (Glushkov Institute of Cybernetics, Ukraine) 
As can be seen from the sensogram (Fig. 1), the shift of resonance angle was higher during the analysis of spermine samples, although the molecular weight of BSA and protein A was higher than polyamines. This was conditioned by the ability of the Plasmontest biosensor device to detect low-molecular weight analytes, and to detect antigen-antibody reaction, which also causes higher angle shift during analysis. The obtained calibration curve will allow checking the presence and determining the content of polyamines in the blood serum of patients with breast cancer. The sensogram shows constant increase in the level of polyamines during the addition of new concentration of polyamines. The sensory properties of the biosensor are not blocked by larger molecules because they are oriented to the detection of low-weight molecules.

After that, the analysis of blood samples of patients with breast cancer was performed. It was determined that in these serums the shift of resonance angle did not exceed the limits of 62.9-63.2 degrees (Fig. 3). As can be seen from the calibration curve, these values correlate with concentration of spermine equaling 5 to $10 \mathrm{ng} / \mathrm{mL}$. In addition, it was observed that the concentration of polyamines correlated with the size of the tumour (Fig. 4). As a result of the studies, it was determined that the concentration of polyamines in blood samples of breast cancer patients was in the limits of $20-100 \mathrm{ng} / \mathrm{mL}$, which exceeds the physiological levels. The concentration of polyamines in the control group did not exceed $7 \mathrm{ng} / \mathrm{mL}$, whereas the concentration of polyamines in group II was in range $21.0-27.2 \mathrm{ng} / \mathrm{mL}$. In Group III the concentration of polyamines was in range 52.5-55.3. However, the concentration of polyamines in blood samples Group IV was in the range 102.3-125.1 ng/mL. According to the results obtained, the developed biosensor method could detect both low and high concentrations of polyamines.

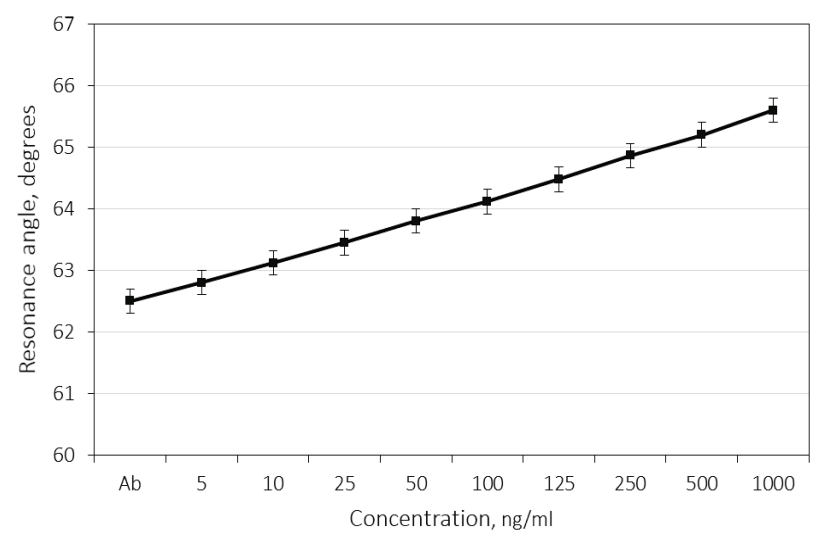

Fig. 2. Resonance angle shifts from concentration of spermine $(5 \mathrm{ng} / \mathrm{mL}-1000 \mathrm{ng} / \mathrm{mL}): \mathrm{x} \pm \mathrm{SD}, \mathrm{n}=5$

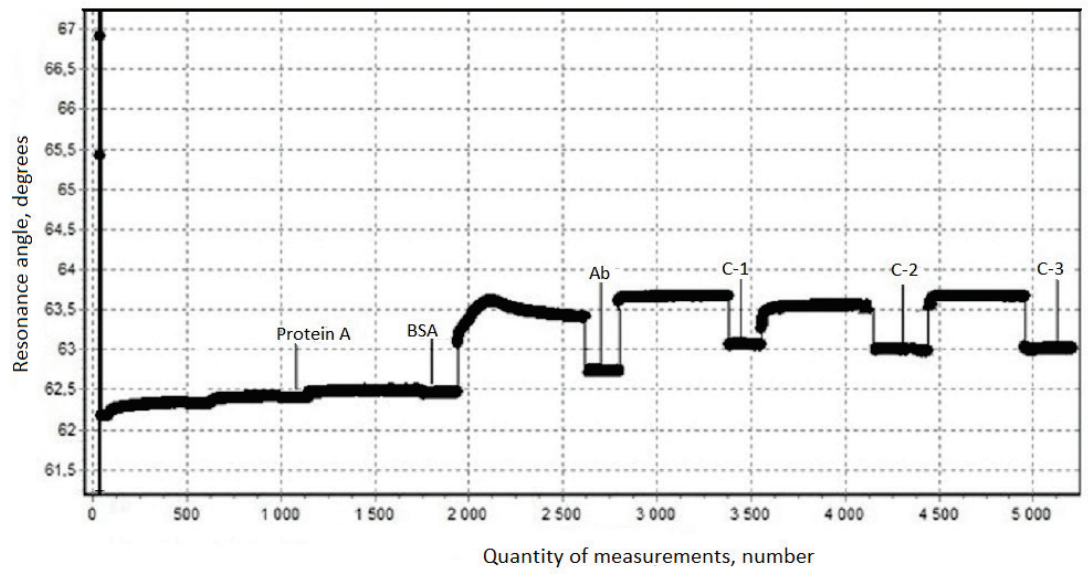

Fig. 3. Sensogram of a biosensor response at different concentrations of spermine in control serum samples 1-3:

BSA - bovine serum albumin; $\mathrm{Ab}$ - anti-spermine serum; C-1, C-2 and C-3 are blood serum samples from the control group

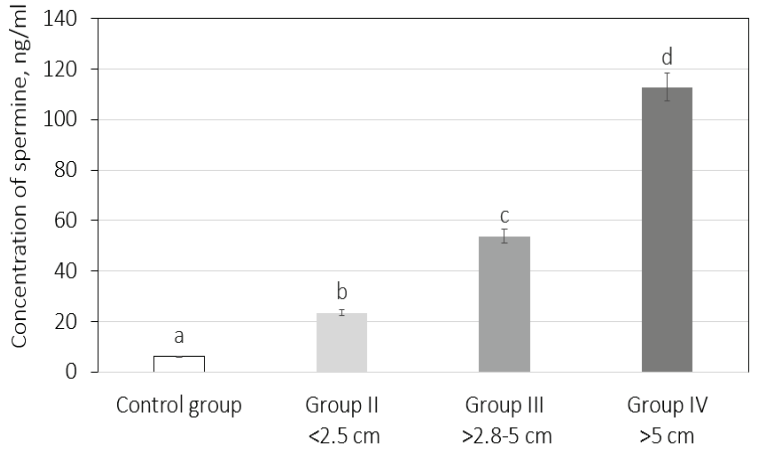

Tumor size, $\mathrm{cm}$

Fig. 4. Concentration of polyamines in the serum of patients with breast cancer depending on the size of the tumour in 4 groups of comparison $(\mathrm{x} \pm \mathrm{SD})$ : different letters indicate the values significantly differing one from another between groups using the Tukey test $(\mathrm{P}<0.05)$ with

Bonferroni correction; control group - healthy persons $(n=9)$;

Group II - patients with tumour size less than $2.5 \mathrm{~cm}(\mathrm{n}=7)$;

Group III - patients with tumour size more than $2.8 \mathrm{~cm}(\mathrm{n}=3)$,

Group IV - patients with tumour size more than $5 \mathrm{~cm}(\mathrm{n}=11)$

It was found that concentration of polyamine was 3.8 times higher in Group II compared with the control, lower by 2.2 times compared with Group III and 4.8 times lower compared with Group IV. Concentration of spermine was 8.8 times higher in Group III compared with
Control group and 2.1 times lower compared with Group IV. Concentration of polyamines in Group IV was 18.5 times higher compared with Control group. As can be seen, the highest concentration of polyamines was detected in samples taken from patients with tumour size more than $5 \mathrm{~cm}$ and concentration of polyamines correlates with tumour size. Therefore, it can be seen that the developed method could detect polyamines in the blood serum of healthy people, as well as of people with disease. In addition, it was also observed that the concentration of polyamines also depends on the age of the patients (Fig. 5).

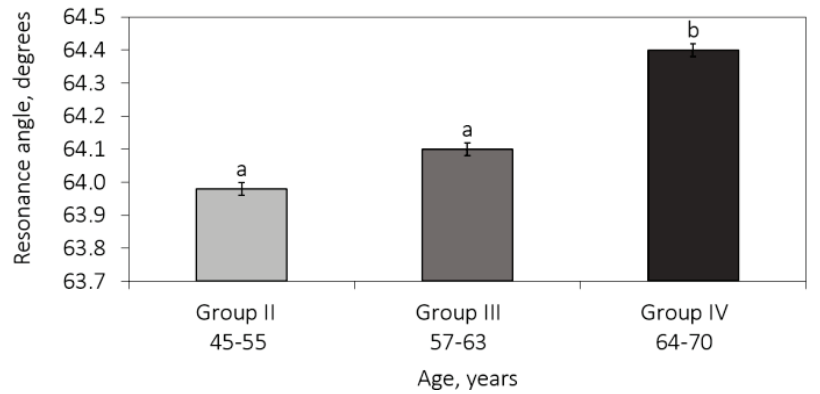

Fig. 5. Resonant angle shifts during analysis of patients different by age: different letters indicate the values significantly differing one from another between groups $(x \pm \mathrm{SD})$ : patients were divided in groups as they were in the previous experiment, except controls; Group II patients aged $45-55$ years $(\mathrm{n}=7)$; Group III - patients aged 57-63 years $(n=3)$, Group IV - patients aged $57-63$ years $(n=11)$ 
The shift of the resonance angle of the biosensor increases according to the age of the patients. Thus, the shift of the resonance angle in samples from patients of Group II aged 45-55 years equaled 63.8-64.1 degrees. In patients in Group III aged 57-63, the shift of the resonance angle was in the range 64-64.2 degrees. In patients in group IV aged 64-70 years, the shift of the resonance angle varied 64.2-64.4 degrees.

According to the data obtained, the resonance angle shift was not only higher in those with larger tumour size but also in older people. Although, significant differences are clearly seen only between groups II and IV. This means that concentration of polyamines depends more on tumour size and stage of disease and less on the age of the patient.

\section{Discussion}

This study was based on the previous research (Prylutskyi \& Starodub, 2016). In this study we described the development of an immunobiosensor testing platform for the express detection of polyamines in breast cancer cells. Detection and estimation of polyamines was made using a special device which was an immunobiosensor working on the basis of surface plasmon resonance effect, where reaction takes place going in real time on the transducer surface, which ends with formation of immune complexes and record of the resonance angle shifts. According to the results obtained in this research, the developed biosensor method could find needed polyamines in very low concentration like $10 \mathrm{ng}$ in $100 \mathrm{~mL}$. Moreover, the correlation of sensitivity of biosensor response to the concentration of polyamines was within $10-100 \mathrm{ng} / \mathrm{mL}$.

The level of major biogenic polyamines in healthy people is in nanomolar concentrations (an average of 4-5 nM). So the concentration of spermine in the blood serum of men is $5.40 \mathrm{nM} / \mathrm{mL}$, and in women is $7.07 \mathrm{nM} / \mathrm{mL}$, concentration of spermidine is $7.54 \mathrm{nM} / \mathrm{mL}$ in men and $9.95 \mathrm{nM} / \mathrm{mL}$ in women, and putrescine $-6.02 \mathrm{nM} / \mathrm{mL}$ in men and $8.32 \mathrm{nM} / \mathrm{mL}$ in women (Minois et al., 2011). Compared with our results the concentration of polyamines in blood samples of breast cancer patients exceeds physiological levels from 3.81 to 18.5 times depending on tumour size. According to other research, the authors observed shifts of resonance angle both after modification of the surface of the transducer and after applying the solutions of polyamines. During the process of immobilization of the sensitive layer, a slight change of resonance angle was observed. For example, during immobilization of antibodies, shifts of resonance angle during the preparation of the sensitive layer were higher for spermine ( 0.06 degrees) than for spermidine ( 0.05 degrees). As it is shown, in general, affinity to antibodies is higher in spermine than in spermidine. In addition, shifts of resonance angle were observed to become higher with the elevation of polyamine concentration. In the course of the study, the obtained data showed the shifts of resonance angle, both after modification of the transducer surface and after applying the solutions of polyamines. This tendency was observed both in the study of spermine and spermidine. During the process of immobilization of sensitive layer, a slight change in resonance angle was observed. Due to the presence of cross-reactivity and lower diagnostic significance of spermidine, in our present research we analyzed spermine (Prilutskiy et al., 2018). In our research we extended the sensitivity of the biosensor to $1000 \mathrm{ng} / \mathrm{mL}$ and did not use spermidine because of the probability of cross-reactivity.

Another research project, which focused on the use of surface plasmon resonance biosensor in diagnostic of breast cancer, reported the possibility of analysis of ErbB2 breast cancer biomarker. An optimization of the analysis was made with signal enhancement and applying of nanoparticles of gold. The gold nanoparticles amplification effect was also studied. The authors performed an essay with clinical trials. The results demonstrated that $50 \%$ of samples, the blood serum contained $180 \mathrm{pg} / \mathrm{mL}$ of polyamines, which was 83 times lower compared with the clinical trials. Lysates, obtained from breast cancer cell lines (SK-BR-3, MCF-7 and MDA-MB-436) have been also estimated. High quantities of the ErbB2 protein were seen in case of overexpression in cell line SK-BR-3. The conducted experiments have proved a high efficiency of SPR biosensor (Eletxigerra et al., 2016).

Research on the detection of biomarkers in body fluids and some tissues using surface plasmon resonance and electroanalytical biosensors has also been conducted. Recently, great impact has been observed from adaptation of new diagnostic methods for timely diagnosing of oncological diseases. The abovementioned researches show some recent developments concerning the surface plasmon resonance technique which was modified for evaluation of breast cancer biomarkers and Alzheimer's disease (AD). Compared with breast cancer, $\mathrm{AD}$ is a neurological disorder related to several biomarkers. SPR biosensors showed many effective properties such as mobility, sensitivity and complex detection of several different samples (Yang et al., 2014). In our research we also used SPRbased biosensor and breast cancer as target disease.

Stojanović et al. (2016) studied the potential abilities of surface plasmon resonance imaging (SPRi) in assessment of apoptotic malignant cells, measuring cytochrome $\mathrm{C}$ excretion. Certain areas on SPR biosensor surface were covered with special anti-cytochrome C, EpCAM and CD49e antibodies. MCF-7 cells were immobilized on the surface of a transducer and put into culture medium which included paclitaxel. Specific shifts on locations covered with EpCAM antibodies were observed, indicating binding with the MCF-7 cells and dependency of the response of the biosensor to presence of the preparation on areas covered with anti-EpCAM, as well as anti-cytochrome C. The researchers made an assumption about the increase in cytochrome $\mathrm{C}$ levels in MCF-7 cells. Also, the obtained results exposed SPRi as a specific method for real time estimation of cancer cells response to drugs or drug combinations (Stojanović et al., 2016). In our research for enhancement of analytical surface we covered the surface of transducer with protein A for better orientation of antibodies and BSA for blocking of unspecific areas.

Monteiro et al. (2015) studied the employment of surface plamon resonance biosensor for evaluation of tumour markers. Studying traditional SPR, however, showed some difficulties in process of experiment. They were eliminated with help of nanohole-based transducers constructed on metal films. The configuration of nanohole arrays showed a specific optical phenomenon. This phenomenon was used in observation of binding processes which occur on metal transducer surface. The authors created a plasmon biosensor based on nanohole transducer created on metal film. The created biosensor could detect a human epidermal receptor protein-2 (HER2) antigen in concentration of $30 \mathrm{ng} / \mathrm{mL}$. (Monteiro et al., 2015). In our research we also used a transducer covered with gold film, but as a target we used polyamines of spermine in blood serum of patients with cancer of the mammary gland.

Kingsnorth et al. examined 54 patients with average age 59.2 years (range 25-82 years). Patients were monitored for two years (range 24 58 months; average - 34.1 months). Eleven women were at pre-menopause. Tumour size was assessed clinically according to the UICC classification. Patients with $\mathrm{T}_{4}$ tumours $(\mathrm{n}=10)$ or with fixed homolateral axillary nodes or with malignant homolateral supraclavicular nodes were treated by a combination of local and systemic therapy.

The polyamines such as spermine, spermidine and putrescine were extracted and separated. Then, their content was expressed in ng. Polyamine levels in breast cancers $(n=54)$ were measured as an additional prognostic marker. The determined concentrations of polyamines (expressed as ng) were in the range of $0.9-4.5 \mathrm{ng}$ for putrescine, 4.2-29.8 $\mathrm{ng}$ for spermidine and 5.6-39.7 ng for spermine. A correlation was observed between the increased intracellular polyamine levels and factors which affect survival after mastectomy, and estrogen-receptor negative status. Increased $\mathrm{T}_{4}$ tumour and medullary-type carcinomas also containned high polyamine levels. Tumour properties do not impact polyamine levels in newly formed tumours. A two year tumour had higher levels of polyamines, compared with a newly formed one. Polyamine concentrations were considered as biological marker of aggressiveness of a tumour and could be used as a prognostic indicator of early tumour recurrence not related to the node status (Kingsnorth et al., 1984). Comparing to the data of the author of the described data, we examined 30 patients different by age and concentration of polyamines. The concentration of spermine was in range of 5-120 ng/ $\mathrm{mL}$ of blood serum.

Other research shows a possibility to detect polyamines in human saliva using ultra-performance liquid chromatography. In this research eight polyamines such as spermine (SPM), cadaverine (CAD) acetylspermine (Ac-SPM), N1-acetylspermidine and N8-acetylspermidine N8Ac-SPD) were analyzed. The correlation the level of polyamines in the 
patients suffering cancer of the mammary gland and the healthy persons was $88 \%$ and $76 \%$ in the preliminary tests, respectively, whereas in those who were on the validation it was about $88 \%$. The authors evaluated the mentioned polyamines in the saliva after performing operation to evaluate the concentration change before and after the treatment. The specificity and sensitivity showed nearly $80 \%$ of the analysis results (Takayama et al., 2016). In our research we analyzed only serum of patients which was enough to evaluate concentration of spermine. Contrary to the conducted studies we were determining free but not acetylated forms of polyamines.

Balcerzak et al. (2017) studied the efficiency of polyamine detection using liquid membrane technology combined with high performance liquid chromatography (HPLC), which appeared to be an appropriate technique to analyze the concentration of polyamines in human body liquids. Determination of polyamines in human body tissues and liquids was used as an additional method. Search for new diagnostic methods is very important. Assessments of the level of polyamines could become this new method. The previous studies have showed that developed technology of polyamine determination could be applied as additional diagnostic tool (Balcerzak et al., 2017). Although the author of that study used HPLC as diagnostic tool, we used SPR-based biosensor and determined that polyamines could be used as a marker in analysis of breast cancer.

Other studies were concentrated on evaluation of polyamines in blood urine and tissue. In cancer tissue, polyamines were significantly higher comparing their levels in controls and in patients. Polyamine concentrations were evaluated using histological assessment and $\mathrm{Ki}-67$ index (Leveque et al., 2000). According to the author, the content of polyamines in the blood of healthy people was $10.1(8.0-12.5) \mathrm{ng} / \mathrm{mL}$. The mean value for spermine equaled $4(3-5) \mathrm{ng} / \mathrm{mL}$. It was revealed that concentration of polyamines in blood serum of healthy people was in range of 5-10 ng/mL, which correlates with the data of the article.

According to the study performed by Byun et al. (2007), HPLC was used for determining the polyamine concentrations in urine and serum obtained from 30 patients with breast cancer, which were compared with 30 normal controls. The obtained results have shown that the polyamine levels in the tissue, urine and serum of patients were significantly higher than in the normal tissue, urine and serum. In comparison, the polyamine levels of patients with breast cancer were higher only in the tissues, and no significant difference in the urine between breast cancer patients and the controls was observed. The analysis of urinary polyamine correlate with the previous studies. Polyamine concentrations determined over the study on mammary gland cancer were lower compared with the prostatic and colonic tissues. Therefore, in the case of breast cancer, the urinary polyamine level would not be a reliable tumour marker in diagnosis or prognosis, unlike in other cancers. In serum, 1,3-diaminopropane, putrescine, spermine, and $\mathrm{N}$-acetylated spermidine levels significantly increased in breast cancer patients. These results correlate well with the previous reports that the serum concentrations of putrescine and spermine in breast cancer patients were higher than in controls. In the study, the serum level of N-acetylated spermidine was also higher in patients suffering breast cancer. According to these results, 1.3-diaminopropane, putrescine, spermine, and Nacetylated spermidine in serum could be potent diagnostic biomarkers of breast cancer (Byun et al., 2007). The results of our research also indicate that in case of cancer of the mammary gland the level of polyamines (spermine in our case) is significantly higher compared with the healthy controls.

Other studies were focused on the analysis of levels of serum polyamine in pre-menopausal breast cancer patients, who were classified as pre- and postsurgery patients and were analyzed. The polyamine concentrations in serum of 58 pre-menopausal breast cancer patients and 45 healthy persons were assessed. Most polyamine levels were higher in pre-menopausal breast cancer patients compared with the controls, except cadaverine and N1-acethylspermine. All polyamine levels were slightly decreased only in postsurgery patients, but not in the controls. A statistical analysis of the level of significance between preand postsurgery periods was performed, and the differences were not significant. The results demonstrated that amount of 1.3- diaminopropane, $\mathrm{N}$-acetylputrescine, $\mathrm{N}$-acetylspermidine, and spermine was higher in presurgery patients compared with the controls. Nacethylputrescine $(\mathrm{P}<0.0005)$ and spermine $(\mathrm{P}<0.005)$ levels were highly increased. Also, serum levels of polyamines were evaluated in 18 patients after menopause and 11 healthy persons of corresponding age. These breast cancer patients were also classified as pre- and postsurgery. In contrast to the data on the pre-menopausal patients, 1.3diaminopropane, putrescine, cadaverine, and $\mathrm{N}$-acetylputrescine levels were higher in post-menopausal breast cancer patients than in the controls. Levels of $\mathrm{N}$-actetylcadaverine, spermidine, $\mathrm{N}$-acetylspermidine, spermine, and N1-acethylspermine were higher in the controls compared with the patients after menopause.

The obtained data demonstrated that the increased polyamine levels in pre-menopausal breast cancer patients return to the levels of the controls after surgery. Moreover, cancer patients did not take any hormones during the experiment, suggesting these results may have no effect on the level of exogenous estrogens. The authors compared both types of patients after and before menopause with the controls. Estimated serum polyamines were higher in breast cancer patients before menopause, while those in post-menopausal patients were similar to the controls. Also, a slight decrease of polyamines in postsurgery patients was observed (Byun et al., 2009). Although in our research we did not focus on the studying pre- and post-menopausal patients, we studied postsurgery patients instead.

According to the research by Persson \& Rosengren (1989), the amount of N1-acetylspermidine was higher in the tumour compared with the small amounts of N1-acetylspermidine found in the normal breast tissues. No N1-acetylspermine was found in the normal breast tissues, and neither the tumour nor the normal breast tissue contained any detectable amounts of N8-acetylspermidine. The amount of free polyamines was also much higher in the tumour tissue than in the normal breast tissue. Elevated levels of N1-acetylspermidine and N1-acetylspermine were observed. Acetylspermidine can be produced by a nuclear acetyltransferase,which also affects a number of other substrates, including histones. However, when spermidine is used as substrate the major product of this enzyme is N1-acetylspermidine and not N1-acetylspermine. Instead, $\mathrm{N} 1$-acetylspermidine is formed by a cytosolic enzyme, namely spermidine/spermine N1-acetyltransferase which exclusively produces N1isomer. This enzyme is highly inducible due to different factors. As for the catabolism, both N1-acetylspermidine and N1-acetylspermine become oxidatively degraded by a polyamine oxidase. The activity of this enzyme greatly exceeds that of the activity of spermidine/spermine N1acetyltransferase, making the acetylation a limiting factor during the process.

According to the data obtained, the acetylating activity of spermidine was much higher in the tumour at presence of N1-acetylspermidine and N1-acetylspermine compared with the normal breast tissue. The lower level of spermidine/spermine N1-acetyltransferase was very low in various tissues and most of the acetylating activity appeared to be performed by other enzymes. Thus, the actual increase in spermidine/spermine N1-acetyltransferase activity is often underestimated. In order to determine whether the increase in acetylating activity of spermidine/ spermine N1-acetyltransferase in tumour tissue was observed, the acetylated products of spermine and spermidine were analyzed. The studies revealed that a large fraction of the synthetized acetylspermidine appeared as N1-acetylspermidine when extracting from the breast cancer tissue. However, a large amount of N1-acetylspermidine was also synthetized, indicating that not all of the acetylating activity was due to the inducible spermidine/spermine N1-acetyltransferase. In contrast, only N8-acetylspermidine was formed in any significant amounts in the extracts from the normal breast tissue. In the context of the fact that some tissues tend to form increased levels of acetylated polyamines, it is interesting is whether these conjugates can be used as markers of growth of tumours (Persson \& Rosengren, 1989). In our research we used not N1acetylspermine, but free spermine contained in blood serum. Our results also showed elevation of polyamine level in cases of tumour presence.

Another study was conducted to analyze the level of polyamines in prostate and breast cancer. A radioimmunoassay was used for determining concentrations of polyamines in plasma. Studies on the sensivity 
was $1 \mathrm{ng}$ of spermine per $100 \mathrm{~mL}$ of plasma and the level of crossreactivity was about $12 \%$ for spermidine and $0.18 \%$ for putrescine. The concentration of spermine in plasma at prostate cancer was measured using radioimmunoassay. The concentrations were only occasionally elevated in patients with prostatic tumours compared with the healthy persons $(0.21 \pm 0.14 \mathrm{ng} / \mathrm{mL})$. A total of $17 \%$ of the patients were observed to have high concentration of polyamines in cases of breast carcinoma. No correlation waso observed between high concentration of polyamines and tumour class or presence of metastases (Chaisiri et al., 1979). Compared with the radioimmunoassay data, biosensor analysis could also detect polyamines in nano concentrations. We did not need additional labels as it was in radioimmunoassay, and we avoided crossreactivity because we did not use other polyamines except spermine.

In another study the levels of polyamines and the other 24 common tumour markers used in clinical practice were studied. The data were obtained by determining the concentrations of every tumour marker of the patients. According to the research, putrescine $(77.7 \%)$ is a more specific marker than spermidine $(59.5 \%)$. Although the specificity of putrescine $(77.9 \%)$ was the lowest, on the other hand, spermine had comparatively excellent specificity $(92.9 \%)$ although the sensitivity $(27.3 \%)$ did not fulfill the expectations. The authors ordered 27 tumour markers in sequence according to the mean values. According to the data obtained, the polyamines, particularly putrescine and spermidine, were more effective than the rest of the tumour markers. These data were useful for clinicians for selecting appropriate markers in the diagnosis of cancers. Also, values of spermine, spermidine, and putrescine as tumour markers in 11 types of cancers were compared. In the case of spermine, the negative predictive value (94.1\%) and total effectiveness $(87.9 \%)$ of pancreas cancer were the highest in 11 types of cancers. Positive predictive value of breast cancer was determined first. In general, over the evaluating of cancer diseases, spermine was much appropriate for diagnosing ovarian cancer because due to its high sensitivity $(48.0 \%)$ and mean value $(74.3 \%)$. In case of spermidine, the negative predictive value (99.2\%) and sensitivity $(94.4 \%)$ in diagnosing pharyngeal cancer were the best for all cancers. Spermidine was suitable for the use at esophageal cancer and pharyngeal cancer. As for putrescine, the negative predictive value and sensitivity were so high that they reached $100 \%$ but the mean value was fourth among 11 types of cancers. Esophageal cancer was the first with the mean value up to $81.9 \%$. Mean values of 27 tumour markers were determined in 11 types of cancers, respectively. Mean values between polyamines were compared with the leading marker of each cancer. The leading marker was the one mean value of which was the highest compared with the other 26 tumour markers in the same type of cancer. Although spermidine and putrescine both adequately reflected the value in esophageal cancer compared with that in other cancers, spermidine $(83.1 \%)$ had higher mean value than putrescine $(81.9 \%)$. The mean values of putrescine were higher compared with spermidine and spermine in most cancers. (Fu et al., 2012). In our study we also used spermine because of its highly specific properties which were shown in our previous research.

In another study, the liquid method of liquid chromatography was used to analyze urinary polyamines and their acetylated metabolites and was proved to be suitable for the quantification of at least nine polyamines such as putrescine (PUT), spermidine (SPD), spermine (SPM), acetylputrescine (AcPUT), acetylcadaverine (AcCAD), N1-acetylspermidine (N1AcSPD), N8-acetylspermidine (N8AcSPD), diacetylspermidine (DiAcSPD) and diacetylspermine (DiAcSPM) from human urine samples, and three polyamines (diaminopropane, cadaverine, acetylspermine) which can be quantified if the concentration in samples is sufficiently high. In addition, two neutral, acetylated diamines can be analyzed semiquantitatively. The parallel analysis of these 14 polyamines was performed without any derivatization using solid phase extraction columns and deuterium labeled internal standards for each analyte in sample preparation. The method appeared to be suitable for the quantification (Häkkinen et al., 2013). Comparing with the presented data, we used SPR-based biosensor and created calibration curve of spermine concentrations. Evaluation of concentration of this polyamine allowed us quantifying the level of polyamines in blood serum. Another study showed the assessment of concentrations of putrescine and cadaverine, in the saliva of healthy persons after waking up and during the day. The authors found putrescine was most abundant compared with cadaverine. After waking up the concentration of polyamines was the highest (putrescine $-33 \mathrm{mg} / \mathrm{mL}$, cadaverine $-17 \mathrm{mg} / \mathrm{mL}$ ) with a decrease during the day (putrescine $-7 \mathrm{mg} / \mathrm{mL}$, cadaverine $-3 \mathrm{mg} / \mathrm{mL}$ ). The authors have developed a new analytical method based on high performance liquid chromatography and fluorescence detection for assessment of amines in human saliva. Sodium fluoride has been shown to be an effective inhibitor of amine formation in saliva at room temperature, allowing samples to be collected and kept without requiring cold (Cooke et al., 2003). Compared with the abovementioned studies, our data represents a possibility to assess the concentration of polyamines in blood serum in nano concentration, which makes our method more effective compared with the data of the abovementioned authors.

In the study described by Fujiwara (1983), an immunoassay was used for the analysis of polyamines using beta-galactosidase labeled spermine and antiserum obtained from rabbits against spermine-BSA. Specificity of anti-spermine serum was determined by measuring the displacement of spermine bound with galactosidase (Spm-fl-Gal) by putrescine, spermidine, and compounds of a similar structure. Calibration curves showing the relationship between the concentrations of unlabeled compounds and the percentage of bound spermine-galactosidase were developed. Experimental conditions for the immunoassay were studied, and it was found that a $0.1 \%$ solution of the antiserum obtained 6 weeks after initial immunization, a $5 \%$ solution of Spm-fl-Gal, $8 \mathrm{~h}$ incubation period with the antiserum with Spm-fl-Gal (in the presence or absence of unlabeled Spm), and a further $3 \mathrm{~h}$ incubation period with a second antibody provided an effective system of analysis at $25{ }^{\circ} \mathrm{C}$. The diluted antiserum bound $55-60 \%$ of the added Spm-fl-Gal in the control tubes, which contained no labeled polyamine and made possible a sensitive test for spermine or spermidine. The sensitivity of both of the assays was defined as the lowest amount distinguishable from zero at the $95 \%$ reliability level was 1 ng per test tube, and the working range was 1-50 ng for each of the polyamines. The reproducibility of the assay was performed within a day (intraassay) and over a period of one week (interassay). The coefficients of variation for intra- and interassays at 5 different levels of spermine in each variation were $7.4-18.1 \%$ and 9.7-27.1\%, respectively. In recovery experiments, 5 normal human sera containing 57.1-65.0 ng of endogenous polyamine per $\mathrm{mL}$ were used. Each serum sample was assessed with and without added spermine $(5,10$, $20,40 \mathrm{ng}$ ), and recovery of the added spermine was calculated from the standard curve. As is shown, the analytical recoveries ranged from 105 $115 \%$. The lowest concentration which was detected with the used method was $1 \mathrm{ng}$ of spermine per test tube. Serum used in the assay exposed $88 \%$ of cross-reactivity to spermidine. No significant cross-reaction comparing with putrescine, diaminopropane, and cadaverine was observed. The authors have developed this method to assess serum levels of polyamines in healthy persons and cancer patients (Fujiwara et al., 1983). In our research we analyzed free polyamines without any additional labels, compared to the research presented in article by Fujiwara. During the experiment, elevation of free spermine levels were observed in the range 5-100 $\mathrm{ng} / \mathrm{mL}$ of blood serum.

\section{Conclusion}

In spite of the results obtained, the following conclusions can be drawn. The SPR-based optical biosensor can detect spermine in the range $5-1000 \mathrm{ng} / \mathrm{mL}$. Calibration curve was developed for further determination of the concentrations of polyamines in blood samples of patients with breast cancer. The level of polyamines was found to correlate with the size of the tumour and the age of the patients with breast cancer. The concentration of polyamines in patients with cancer was in the range 21.3$125.1 \mathrm{ng} / \mathrm{mL}$, compared with the control samples from healthy people, in which the level of polyamines did not exceed 5.3-7.0 ng/mL. In addition, the study revealed that the shift of the resonance angle of the biosensor during comparative analysis of different age groups gradually increases with increasing of the age of patients, but also depends on the size of the tumour. It turned out that the shift of the resonance angle increases in patients with an increase of the tumour size. 
Study was conducted with the help of Plasmontest biosensor device given by Glushkov Institute of Cybernetics, Kyiv, Ukraine. The samples of blood serum were provided by “Alpha-Lab Service” Diagnostic Laboratory, Kharkiv, Ukraine.

\section{References}

Bae, D. H., Lane, D. J., Jansson, P. J., \& Richardson, D. R. (2018). The old and new biochemistry of polyamines. Biochimica et Biophysica Acta, 1862(9), 2053-2068.

Balcerzak, W., Pokajewicz, K., \& Wieczorek, P. P. (2017). A useful procedure for detection of polyamines in biological samples as a potential diagnostic tool in cancer diagnosis. Applied Cancer Research, 37(1), 1-7.

Byun, J. A., Choi, M. H., Moon, M. H., Kong, G., \& Chul Chung, B. (2009). Serum polyamines in pre- and post-operative patients with breast cancer corrected by menopausal status. Cancer Letters, 273(2), 300-304.

Byun, J. A., Lee, S. H., Jung, B. H., Choi, M. H., Moon, M. H., \& Chung, B. C. (2007). Analysis of polyamines as carbamoyl derivatives in urine and serum by liquid chromatography - tandem mass spectrometry. Biomedical Chromatography, 22(1), 73-80.

Casero, J. R., Murray, T. S., \& Pegg, A. E. (2018). Polyamine metabolism and cancer: Treatments, challenges and opportunities. Nature Reviews Cancer, 18(9), 681-695.

Cervelli, M., Angelucci, E., Germani, F., Amendola, R., \& Mariottini, P. (2013). Inflammation, carcinogenesis and neurodegeneration studies in transgenic animal models for polyamine research. Amino Acids, 46(3), 521-530.

Chaisiri, P., Harper, M. E., \& Griffiths, K. (1979). Plasma spermine concentrations of patients with benign and malignant tumours of the breast or prostate. Clinica Chimica Acta, 92(2), 273-282.

Cooke, M., Leeves, N., \& White, C. (2003). Time profile of putrescine, cadaverine, indole and skatole in human saliva. Archives of Oral Biology, 48(4), 323-327.

Damiani, E., \& Wallace, H. M. (2017). Methods in molecular biology. Humana Press, New York

Eletxigerra, U., Martinez-Perdiguero, J., Barderas, R., Pingarrón, J. M., Campuzano, S., \& Merino, S. (2016). Surface plasmon resonance immunosensor for ErbB2 breast cancer biomarker determination in human serum and raw cancer cell lysates. Analytica Chimica Acta, 905(4), 156-162.

Fu, S., Xiao, C., Zhao, W., \& Yu, X. (2012). Polyamines analysis by HPLC and their application as tumor markers. Frontiers in Bioscience, 4(5), 1795-1801.

Fujiwara, K., Asada, H., Kitagawa, T., Yamamoto, K., Ito, T., Tsuchiya, R., Sohda, M., Nakamura, N., Hara, K., Tomonaga, Y., Ichimaru, M., Takahashi, S. (1983). Preparation of polyamine antibody and its use in enzyme immunoassay of spermine and spermidine with $\beta$-D-galactosidase as a label. Journal of Immunological Methods, 61(2), 217-226.

Häkkinen, M. R., Roine, A., Auriola, S., Tuokko, A., Veskimäe, E., Keinänen, T. A., Lehtimäki, T., Oksala, N., \& Vepsäläinen, J. (2013). Analysis of free, mono- and diacetylated polyamines from human urine by LC-MS/MS Journal of Chromatography B, 941(23), 81-89.

Kingsnorth, A. N., Wallace, H. M., Bundred, N. J., \& Dixon, J. M. J. (1984). Polyamines in breast cancer. British Journal of Surgery, 71(5), 352-356.

Kiran, K., Kumar, S., \& Singh, R. (2018). Tumour marker and diagnosis of cancer. Indian Journal of Forensic Medicine and Toxicology, 12(3), 14-20.

Levêque, J., Levêque, J., Foucher, F., Bansard, J.-Y., Havouis, R., Grall, J.-Y., \& Moulinoux, J.-P. (2000). Polyamine profiles in tumor, normal tissue of the homologous breast, blood, and urine of breast cancer sufferers. Breast Cancer Research and Treatment, 60(2), 99-105.

Mandal, S., Mandal, A., Johansson, H. E., Orjalo, A. V., \& Park, M. H. (2013). Depletion of cellular polyamines, spermidine and spermine, causes a total arrest in translation and growth in mammalian cells. Proceedings of the $\mathrm{Na}$ tional Academy of Sciences, 110(6), 2169-2174.

Minois, N., Carmona-Gutierrez, D., \& Madeo, F. (2011). Polyamines in aging and disease. Aging, 3(8), 716-732.

Monteiro, J. P., de Oliveira, J. H., Radovanovic, E., Brolo, A. G., \& Girotto, E. M (2015). Microfluidic plasmonic biosensor for breast cancer antigen detection. Plasmonics, 11(1), 45-51.

Moskalets, A. I., \& Shcherbina, O. V. (2011). Opuholevyie markeryi v laboratornoy diagnostike [Tumor markers in laboratory diagnostics]. Laboratory diagnostics, 55(1), 64-72 (in Russian)

Park, M. H., \& Igarashi, K. (2013). Polyamines and their metabolites as diagnostic markers of human diseases. Biomolecules and Therapeutics, 21(1), 1-9.

Persson, L., \& Rosengren, E. (1989). Increased formation of N1-acetylspermidine in human breast cancer. Cancer Letters, 45(2), 83-86.

Prylutskyi, M., Starodub, N., \& Bilko, N. (2016). Determination of the concentration of polyamines with SPR-based immune biosensor for early diagnostics of breast cancer. Journal of Biosensors and Bioelectronics, 7(4), 1-4.

Prylutskyi, M., Starodub, N., Lebyedyeva, T., \& Shpylovyy, P. (2018). Express control of levels of polyamines by immune biosensor based on SPR. Bulletin of Taras Shevchenko National University of Kyiv, 25(2), 59-63.

Soda, K., Dobashi, Y., Kano, Y., Tsujinaka, S., \& Konishi, F. (2009). Polyaminerich food decreases age-associated pathology and mortality in aged mice. Experimental Gerontology, 44(11), 727-732.

Stojanović, I., van Hal, Y., van der Velden, T. J. G., Schasfoort, R. B. M., \& Terstappen, L. W. M. M. (2016). Detection of apoptosis in cancer cell lines using surface plasmon resonance imaging. Sensing and Bio-Sensing Research, $7(1), 48-54$.

Takayama, T., Tsutsui, H., Shimizu, I., Toyama, T., Yoshimoto, N., Endo, Y., Toyo'oka, T. (2016). Diagnostic approach to breast cancer patients based on target metabolomics in saliva by liquid chromatography with tandem mass spectrometry. Clinica Chimica Acta, 452, 18-26.

Yang, M., Yi, X, Wang, J, \& Zhou, F. (2014). Electroanalytical and surface plasmon resonance sensors for detection of breast cancer and Alzheimer's disease biomarkers in cells and body fluids. The Analyst, 139(8), 1814-1825

Yatsenko, L. D. (2014). Rol' biomarkerov v patogeneze zlokachestvennyih obrazovaniy [Role of biomarkers in pathogenesis of malignant rejuvenescence]. World of Medicine and Biology, 43(1), 192-195 (in Russian). 